\title{
A human vision based system for biometric images recognition
}

\author{
Wassila Boukhari, Mohamed Benyettou, Belmadani Abderrahim \\ Département d'informatique, Faculté des Mathématiques et de l'informatique, Université des Sciences et de la Technologie d'Oran \\ Mohamed Boudiaf, Oran, Algérie
}

\begin{tabular}{l} 
Article Info \\
\hline Article history: \\
Received Oct 7, 2021 \\
Revised Dec 24, 2021 \\
Accepted Jan 17, 2022 \\
\hline
\end{tabular}

Keywords:

Biometric

Frequency filtering

Human vision

Input coding

Liquid state machine

\begin{abstract}
In this paper, a universal biometric system based on human vision is proposed. From recent biological and physiological results, A human identification system that approximates the natural vision and recognition of individuals is conceived. Liquid state machine (LSM), as a recurrent spiking neural network, is highly inspired by the brain neural architecture with low training cost. However, input dimension of large scale images requires efficient processing at the cost of performance or resource overhead. This paper propose a new neural input coding for images based on frequency signals rather than pixels. Each image is filtered and fragmented then the LSM liquid (or reservoir) will receive, first, high frequency signals, then low frequency signals from each fragment. The two sets of output neurons states corresponding to each type of filter will be matched to the entire enrollment database. A weighted sum rule between the matching results will determine the right class of a biometric image. The system was tested on three different biometric datasets: face, palmprint and off-line signature, results show the reliability of the proposed approach.
\end{abstract}

This is an open access article under the CC BY-SA license.

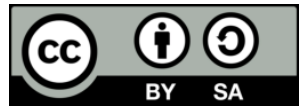

\section{Corresponding Author:}

Wassila Boukhari

Department of Computer Science, Faculté des Mathématiques et de l'informatique

Université des Sciences et de la Technologie d'Oran Mohamed Boudiaf

BP 1505, El M'naouer, 31000, Oran, Algérie

Email: wassila.boukhari@univ-usto.dz

\section{INTRODUCTION}

Based on biological and behavioral traits as face, voice, hand geometry, keystroke, iris or fingerprints; biometric came as the most pertinent solution to document fraud and identity theft by authenticating and identifying individuals in a reliable and fast way. Biometrics allow a person to be identified based on a set of recognizable and verifiable data which are unique and specific to him. Person physiological traits, which are enrolled by means of an image capture device, including face, iris, hand or fingerprints are the most commonly used biometrics. Since each of these images is different in type, structure and size; a specific image preprocessing method would be more appropriate for one specific biometrics than the others. Therefore, a biometric system is generally conceived for a specific image; even when this system is multimodal, each modality needs a specific preprocessing scheme. However, the biological vision system is able to process images of different sizes and structures in the same manner. Two functional parts are involved in human vision, the eye and about $50 \%$ of the brain. While the eye functions as biological equivalent of camera, the brain does all of the complex image processing.

Processing by the brain consists of partly of simple image processing and partly of higher functions which build and manipulate an internal model of the outside world. Artificial neural networks (ANNs) [1] are a variety of models which simulate brain neural information processing. ANNs have been used successfully in many real world applications including image processing and pattern recognition [2]. The closet ANN 
architecture to the brain structure is the recurrent neural network (RNN) model. RNNs are dynamical systems with temporal state representation; they are computationally powerful and can be used in many temporal processing and applications. However, RNNs training algorithms based on direct optimization of the network weights have led to less than satisfactory results, they usually exhibit slow convergence and high computational requirements.

Reservoir computing (RC) is a novel approach proposed by Jaeger in [3] that can cope with realtime computations on recurrent neural networks without the constraint of complex training. The main idea of $\mathrm{RC}$ is to use a large but fixed recurrent part as a reservoir of dynamic features and to train only the output layer to extract the desired information. The liquid state machine (LSM) [4] is a recurrent network with a dynamic reservoir using spiking neurons connected by dynamic synapses. The reservoir (or liquid) in the LSM which represents temporal dependencies does not have to be trained, which therefore makes the learning process fast and simple. Furthermore, the LSM bears many similarities to biological neural networks and can therefore profit from new findings in neurobiology. It has been used for various real world applications such as movement prediction [5], [6], speech recognition [7]-[9], video activity and image recognition [10]-[12] and was particularly successful used in spatio-temporal pattern recognition [13]-[16].

To cope with the state-of-the-art results in real world applications, many efforts on enhancing the LSM performance were based on the exploration of different LSM topologies [17], [18], new training algorithms or cost-intensive parameters search [19], [20], which increase accuracy at the cost of performance or resource overhead. The input format is another issue that has significant impact on the performance of the LSM. In general, the number of input pixels represents the number of the input layer neurons. Processing large images is more time-consuming and resource demanding than a small input given the same liquid architecture and parameters. Some previous studies proposed different methods for encoding the input based on temporal encoding [21] or rate codes [22], but with the increase in the size of images, the converted input size is also growing rapidly to a large scale. Recently, a study using a different image coding based on prolate spheroidal wave functions (PSWF) [23] gave significant results on the modified national institute of standards and technology (MNIST) digit database.

Based on the significant separation property of the LSM which assumes that different inputs to a pool of neurons; which represents the liquid or reservoir of the LSM, should cause different neuron responses; and similar inputs should produce same responses [24], the successful LSM results obtained dealing with analogue signals as inputs and finally the experimental results in biology and neurophysiology which proved that the visual system analyses inputs in several spatial resolution scales; which motivated the use of spatial frequency preprocessing of images [25]-[27] in computer vision and pattern recognition, we propose a universal human based biometric identification system using the LSM with its biologically inspired parameters and structure with a new image encoding method using, rather than pixels, an analogue signal obtained by mapping a frequency filtered image. To increase furthermore the efficiency of the proposed system, every input image is splitted in 16 fragments. The input layer is then composed of 16 channels (neurons) that will receive, at the same time, an image fragments signal. The responses collected from the reservoir neurons represent the image code for classification. The same system architecture and parameters was tested on three different biometric datasets: face, palmprint and off-line signature with significant and competitive results.

\section{MATERIALS}

\subsection{Filtering images in frequency domain}

According to Sinha et al. [28], both high and low frequency information are used in the human recognition task. A simple mathematical transformation algorithm such as fourier transform (FTT) or discrete cosine transform (DCT) can change an image from pixels to frequencies. Then, with an appropriate filter one can separate low frequencies from high frequencies to perform image analysis and pattern extraction.

In spatial frequency analysis, an image is represented as a weighted combination of basic functions, in which high frequencies carry finely, detailed information and low frequencies carry coarse, shape-based information. Filtering with fast fourier transform (FFT) is one of the techniques used to emphasis the low and high spatial frequency components. Assume $\mathrm{I}(\mathrm{m}, \mathrm{n})$ is a $\mathrm{M} \times \mathrm{N}$ image. Let $\mathrm{F}(\mathrm{k}, \mathrm{l})$ denote the 2DFFT of the images $I(m, n)$, which is given by (1):

$$
\begin{aligned}
& F(k, l)=\sum_{m=0}^{M-1} \sum_{n=0}^{N-1} I(m, n) e^{-j 2 \pi\left(\frac{m k}{M}+\frac{n l}{N}\right)} \\
& =A_{F}(k, l) e^{j \theta F(k, l)}
\end{aligned}
$$


where $\operatorname{AF}(k, l)$ is magnitude components, and $\theta F(k, l)$ is phase components. $F$ is called the spectrum of the image I, let $\mathrm{H}$ and $\mathrm{G}$ denote the filter transfer function, and the spectrum of the filtered image I', respectively. The convolution theorem states that:

$$
\mathrm{G}(\mathrm{k}, \mathrm{l})=\mathrm{F}(\mathrm{k}, \mathrm{l}) * \mathrm{H}(\mathrm{k}, \mathrm{l})
$$

The filtered image I' can be computed using the inverse FFT. Generally, most filters do not affect phases and change only magnitude. A high pass filtering suppresses low frequencies components and produces images with enhanced edges while low pass filtering suppresses high frequency components and produces smoothed images. Figure 1(a) give an example of an orginal image before filtering while Figure 1(b) and (c) illustrate the effects of high pass filtering and low pass filtering on this image respectively.

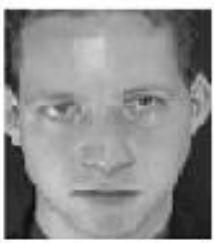

(a)

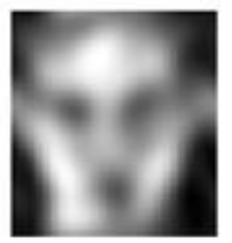

(b)

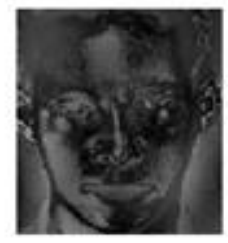

(c)

Figure 1. Image filtering in frequency domain, (a) original image, (b) high frequencies image,

(c) low frequencies image

\subsection{The liquid state machine}

Introduced by Maass [29], the LSM was originally presented as a framework for analysis of realtime computation on continuous input time series. Highly inspired by brain microcircuits, the LSM is composed of randomly connected spiking neurons created using biologically inspired parameters and excited by external input spike trains. Theoretically spiking neurons are computationally powerful as they are able to react non-linearly to individually timed inputs. Consequently, the liquid state machine is a very powerful model. The LSM does not require a task-dependent construction of a neural network and comprised essentially three parts as shown in Figure 2, an input layer U, a large randomly connected core LM (the dynamic reservoir or liquid) which has the intermediate states transformed from inputs, and an output layer FM, a reading card, that allows extracting from the network states XM at a given time an information determined by learning.

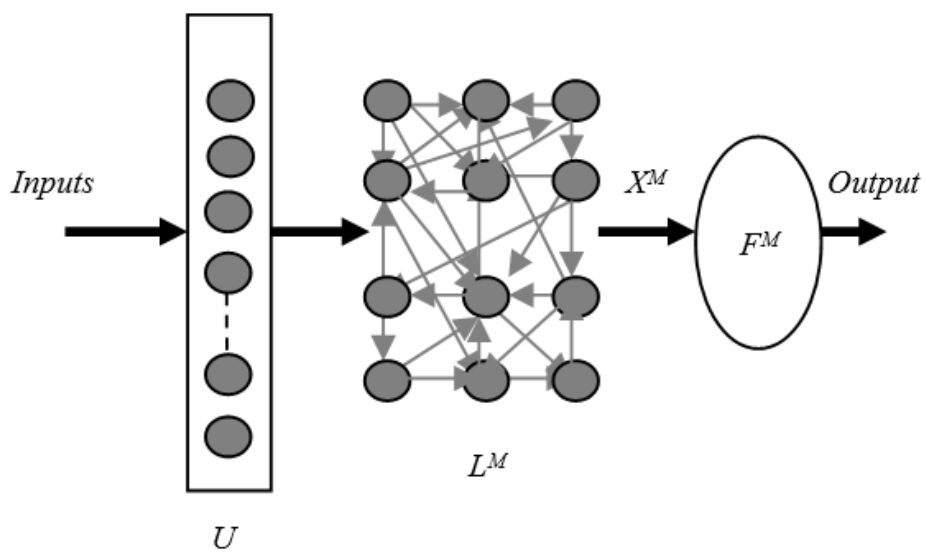

Figure 2. LSM architecture

In our experiments we construct a liquid having the same architecture proposed in [29] with 135 spiking neurons, shaped like a column of 3 by 3 by 15 neurons randomly connected, the input unit in our case is composed of 16 neurons. Instead of training such a complex architecture of the liquid, Maass [29], 
introduced the so-called separation propriety (SP) of the liquid. SP addresses the ability to separate two different input sequences from each other, this is important because the readout network needs to be able to separate two input patterns to have a good performance. If two patterns look too much alike if they should not, the readout network can not differentiate between the two patterns and thus is not able to tell which pattern belongs to which class. The other property of the liquid is the approximation property (AP) which addresses the ability of the readout network to distinguish two different patterns and transform the states of the liquid into the given target output.

The function of the liquid is to project inputs into a high dimensional intermediate state allowing the outputs to be linearly separable. The liquid state is simply the current output of some operator or filter LM that maps input functions $\mathrm{u}($.) onto functions $\mathrm{XM}(\mathrm{t})$. A basic description of the LSM method is given by the following:

$$
\begin{aligned}
& X^{M}(t)=\left(L^{M} u\right)(t) \\
& y(t)=F^{M}\left(X^{M}(t)\right)
\end{aligned}
$$

in (4) computes the output $y(t)$ by applying a readout map function $\mathrm{FM}(\mathrm{t})$ to the current liquid state $\mathrm{XM}(\mathrm{t})$. The readout map FM structure is not given explicitly and all available statistical analysis or pattern recognition methods can be used.

\section{METHOD}

The proposed system performs biometric identification by following a series of steps as shown in Figure 3. The preprocessing step consists of separating high frequencies from low frequencies in the given image resulting into two subimages: HF image which contains only high frequencies and LF image with low frequencies. Then, both HF and LH are fractioned into 16 equally sized parts. Mapping each part produces a frequency signal going to a specific channel of the input unit as shown in Figure 4.

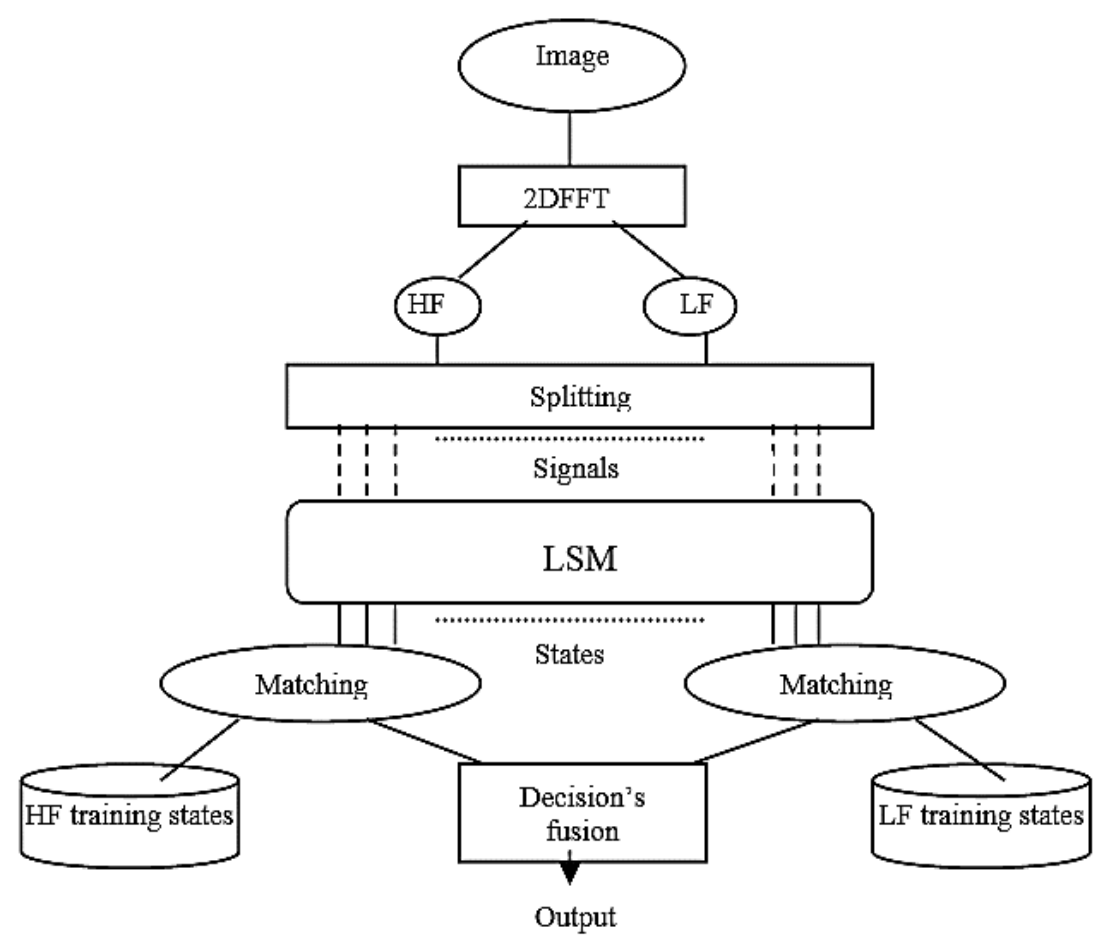

Figure 3. Architecture of the proposed recognition system 


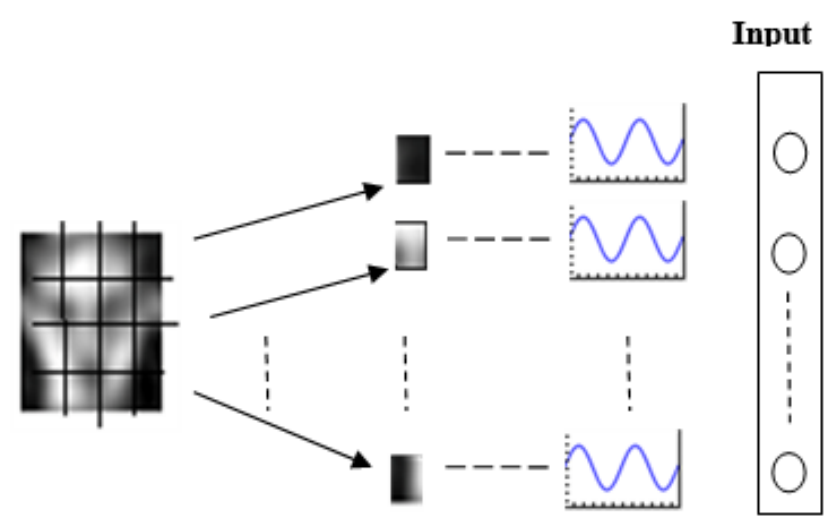

Figure 4. Input image coding

There will be two state vectors for every input image. Let X1 and X2 denote the state vectors resulting from HF simulation and LF simulation respectively. The training states databases are created by recording all the state vectors of the training set samples. A matching process between both X1 and X2 with their corresponding training database will determine two different or similar targets; a fusion algorithm will be applied on these two outputs to determine the finale decision.

Determining the simulation target output is the role of the FM unit (Figure 2). Since there is no specific algorithm assigned to this unit, many pattern classification methods have been used to classify a state vector XM. In his original paper, Maass [29] used a simple Euclidian distance to measure the separation property of the LSM, similar geometric distance between the centroïds of the states that belong to different classes is used in [30] and a feed forward neural network in [31]. In our experiments, a simple Hamming distance described in (5) is used to compare between state vectors:

$$
\mathrm{D}(\mathrm{x}, \mathrm{y})=\sum_{\mathrm{k}=0}^{\mathrm{n}-1}\left[\mathrm{x}_{\mathrm{k}} \neq \mathrm{y}_{\mathrm{k}}\right] / \mathrm{n}
$$

where $\mathrm{x}, \mathrm{y}$ is two vector of size $\mathrm{n}$, the hamming distance calculate the percentage of coordinates that differ between $\mathrm{x}$ and $\mathrm{y}$. For the best matching, the Hamming distance should be zero.

A double simulation of the LSM is performed, first with high frequencies images of each person then with low frequencies images resulting into two given results. The right decision is then obtained by fusion using the weighted sum rule algorithm described in the following steps. Compare X1 and X2 with registration databases (see section 2.3), let R1 and R2 the hamming distance vectors obtained for X1 and X2 respectively. $\mathrm{R}$ is the weighted sum of $\mathrm{R} 1$ and $\mathrm{R} 2$ as described in (6):

$$
\mathrm{R}=\mathrm{w}_{1} * \mathrm{R} 1+\mathrm{w}_{2} * \mathrm{R} 2
$$

where w1, w2 are associated weights for $\mathrm{R} 1$ and $\mathrm{R} 2$ respectively. The minimum distance in $\mathrm{R}$ will determine the right class of the input image.

\section{RESULTS AND DISCUSSION}

This paper presents a universal biometric system based on human vision. With nearly the same input parameters, this system should work for different biometric data. To evaluate the performance of the proposed method, experiments were conducted on three famous databases: the ORL face database, the PolyU palmprint database and GPDS off-line signature database.

\subsection{The ORL database}

the ORL face database was conceived at the Olivetti Research Laboratory in Cambridge University, UK. The ORL database is composed of 400 face images taken from 40 different persons (4 female and 36 male) captured over the span of a 2-year period from subjects aged between 18 to 81 . The total samples for each individual is 10. All images are different in term of position of the face, rotation, scale and expression. For some persons, the images were taken at different time, varying facial details (glasses / no glasses). Also, the images were manually cropped and rescaled to a dimension of 112x92, 8-bits grey levels. Examples of the ORL image samples are given in Figure 5. 

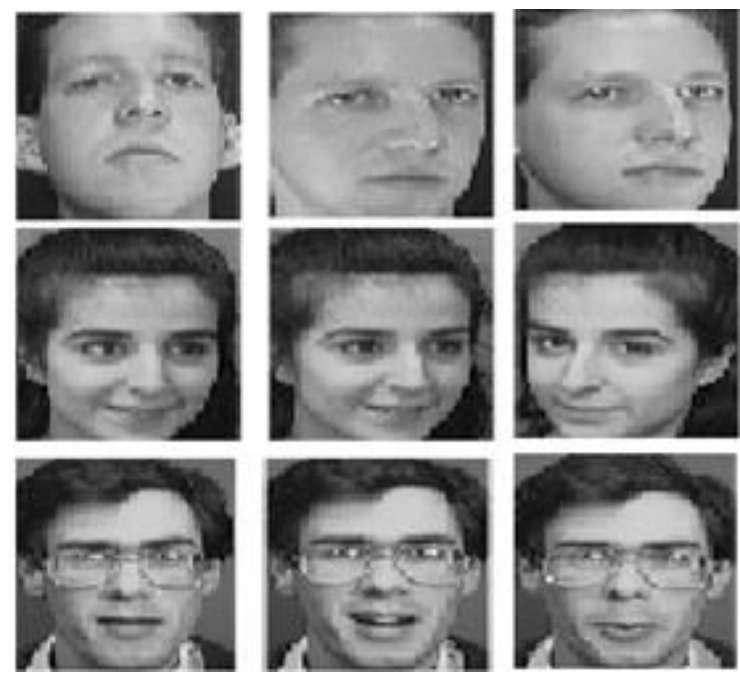

Figure 5. Face samples from ORL database

\subsection{The PolyU palmprints database}

We made use of the hong kong polytechnic university (PolyU) palmprint database version II to evaluate the effeciency of our approach. We took out 1000 palmprint greyscale images from this database of 100 different individuals with 10 samples for each person. Each experiment was setup on two registration databases with different number of palms, the first database contains 500 images from 50 different palms and the second registration database has 1000 templates from 100 different palms. In this database, the central part of each original image was automatically cropped using the algorithm mentioned in [32]. The cropped images were resized to $128 \times 128$ pixels and pre-processed by histogram equalization. Figure 6 shows some sample images of two palms in the database.
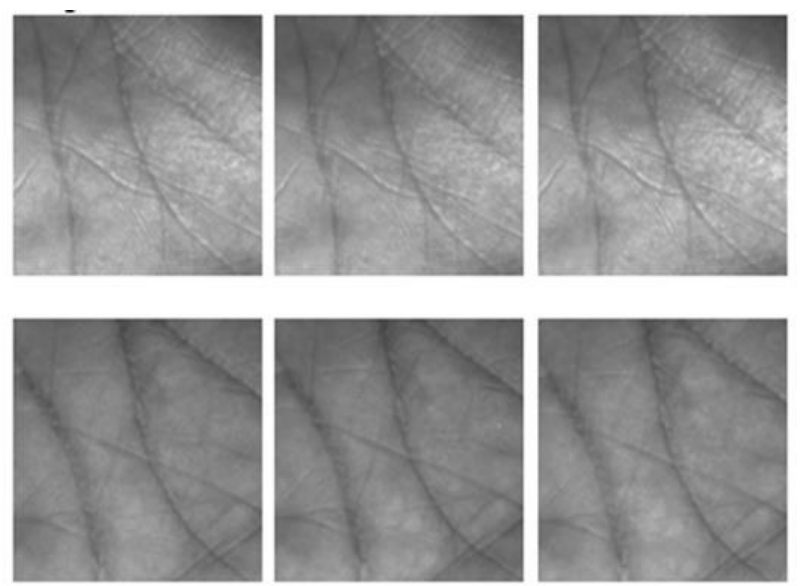

Figure 6. Sample images in the PolyU palmprint database

\subsection{The GPDS off-line signature database}

The results on off-line signatures images given in this paper are obtained using the "Grupo de Procesado Digital de Senales" (GPDS) signature database. We used a total of 210 signatures in this research. Those 210 signatures are comprised of 30 sets (i.e. from 30 different people) and, for each person there are 7 samples of genuine signatures. Figure 7 shows some of the signatures in the GPDS database, three samples from the same person are given in Figure 7(a) and three signatures of an other person are given in Figure 7(b). On each experiment, different training and testing set sizes were adopted to evaluate the system accuracy. 


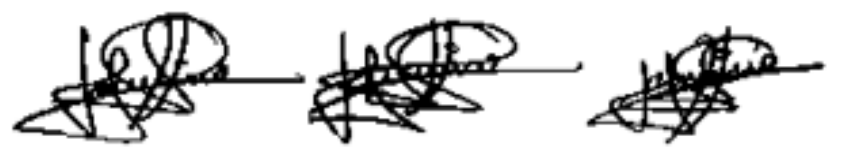

(a)

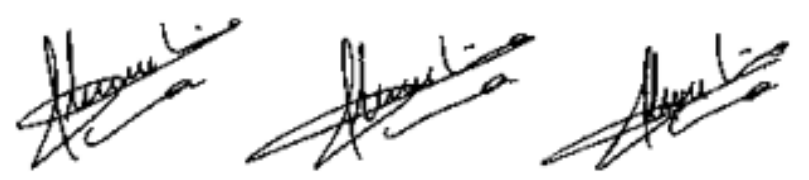

(b)

Figure 7. Signatures from two different persons from the GPDS database, (a) samples of the first person and (b) samples of the second person

\subsection{Experiments on the ORL database}

To evaluate the performance of the proposed system on face images, two different experiments were conducted. In the first experiment, a fixed set of images is used for training while the remaining samples are used for testing. The recognition rate is calculated varying the size of the training set from one up to six samples (Table 1). The second experiment consist of calculating the average recognition rate among different combinations of different images in the training set (Table 2). For example, in the case of three samples in the training set and seven samples in the testing set; each time a different combination of three images of a given face is taken for training; the evaluation is done on the remaining images and the maximum score of each person is enrolled. The accuracy of the system is then given by calculating the average score among all combinations. Table 1 and Table 2 show the obtained results of first and second experiment respectively compared to other benchmarked face recognition algorithm.

Table1. Performance comparison on the ORL database for different numbers of deterministic training samples per class

\begin{tabular}{lcccccc}
\hline \multirow{2}{*}{ Methods } & \multicolumn{7}{c}{ Recognition rates $(\%)$} \\
& $\mathrm{r}=1$ & 2 & 3 & 4 & 5 & 6 \\
\hline PCA & - & 71 & 73 & 77 & 78 & 89 \\
LDA & - & 78 & 82 & 87 & 87 & 93 \\
PCA-NN & - & 75 & 76 & 80 & 85 & 90 \\
LDA-NN & - & 80 & 84 & 89 & 91 & 93 \\
HLSM & 68.65 & 73.75 & 80 & 83.75 & 85 & 89.38 \\
LLSM & 50.83 & 62.19 & 74.29 & 75.83 & 81 & 83.75 \\
FLSM & 75.90 & 84.38 & 89.64 & 93.75 & 96 & 98.75 \\
\hline
\end{tabular}

Table 2. Results on the ORL database under different numbers and combinations of the training samples

\begin{tabular}{lcccccc}
\hline \multirow{2}{*}{ Methods } & \multicolumn{6}{c}{ Average recognition rates (\%) } \\
& $\mathrm{r}=1$ & 2 & 3 & 4 & 5 & 6 \\
\hline RBFNN & - & 86.31 & 93.86 & 96.25 & 97.30 & - \\
HLSM & 80.95 & 90 & 92.50 & 94.58 & 94.50 & 95 \\
LLSM & 67.27 & 82.19 & 87.50 & 92.08 & 93 & 95 \\
FLSM & 86.75 & 96.25 & 96.43 & 98.33 & 98.50 & 99.38 \\
\hline
\end{tabular}

For both experiments on the ORL database, results of high frequencies simulations (HLSM), low frequencies simulations (LLSM) and the fusion decision (FLSM) are reported on Table 1 and Table 2. When the training set was deterministic, the highest result (98.75\%) was obtained by the FLSM method. Compared to popular face recognition approaches as principal component analysis (PCA) or linear discriminant analysis (LDA) with a recognition rate of $89 \%$ and $93 \%$ respectively, the proposed approach gives best results. A neural network associated with PCA and LDA [33] performs better at the recognition level (90\% and 93\% respectively), but remains outperformed by the proposed method $(98.75 \%)$. The second experiment was made by a randomly selected training templates (Table 2), the average recognition rates (ARR) of the HLSM and LLSM with a training set of six images are approximately the same (95\%), when the FLSM approach obtained the best results with an ARR of $99.38 \%$. A radial basis function neural network (RBFNN) method 
for face recognition proposed in [34] is used for comparison. The ARR of the RBFNN method with five training templates is $97.30 \%$ which is outperformed by the FLSM ARR $(98.50 \%)$ with the same training set size.

\subsection{Experiments on the PolyU database}

Different numbers of training and testing templates on the two palmprint registration databases were taken, with no overlap between training and testing sets. Since each palm has 10 different templates, three of them have been used as testing templates at the first time then the size of the testing set changes according to the number of training templates. Each of the palmprint images in the testing database is matched with all of the palmprint images in the registration database to generate incorrect and correct identification. A summary of the identification tests results is given in Table 3 with $\mathrm{N}=50$ and $\mathrm{N}=100$.

Table 3. Results on the PolyU database $(\mathrm{N}=50$ and $\mathrm{N}=100)$ for different numbers of training samples ( $\mathrm{r}$ ) and test samples $(\mathrm{k})$ per class

\begin{tabular}{|c|c|c|c|c|}
\hline \multirow{2}{*}{ Databases } & \multirow{2}{*}{ Test samples } & \multicolumn{3}{|c|}{ Recognition rates $(\%)$} \\
\hline & & $\mathrm{r}=1$ & $r=2$ & $r=3$ \\
\hline \multirow[t]{2}{*}{$\mathrm{N}=50$} & $\mathrm{k}=10-($ training samples $)$ & 92.22 & 97.75 & 99.71 \\
\hline & $\mathrm{k}=3$ & 91 & 96 & 98.33 \\
\hline $\mathrm{N}=100$ & $\mathrm{k}=10-($ training samples $)$ & 85.56 & 92.88 & 96 \\
\hline
\end{tabular}

The results show that increasing $\mathrm{N}$ is equivalent to decreasing the accuracy of the system. A high recognition rate $(99.71 \%)$ was obtained using the first registration database $(\mathrm{N}=50)$ with only three training samples and seven testing images which is a competitive result compared to other palmprint recognition methods like in [35], the same experiment on the second database $(\mathrm{N}=100)$ results in a reasonable accuracy of $98.33 \%$ when the number of testing samples was three and $96 \%$ with seven templates as testing images.

\subsection{Experiments on the off-line signatures GPDS database}

Experiments on the GPDS database were made by changing the size of the gallery $(10,20$ and 30 persons) and the training set (2, 3 and 4 signature templates). Figure 8 shows the obtained recognition rate on each experiment. When the registration database comprised only ten person's signatures, the highest RR $(96.67 \%)$ was obtained with four training templates, while with the same training set size the second registration database (20 persons) and the third database (30 persons) obtained an RR of 93.33\% and $85.60 \%$ respectively.

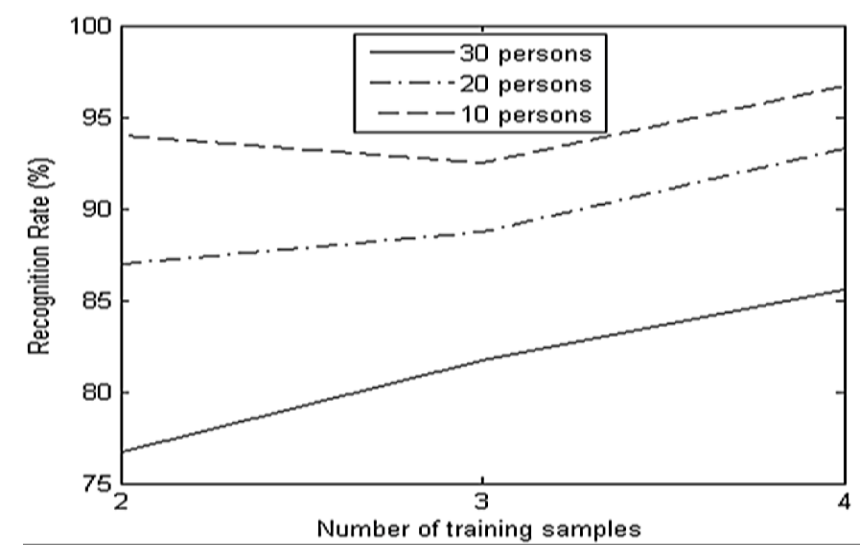

Figure 8. Recognition rates (RR) of the proposed system on the GPDS database

\section{CONCLUSION}

In this paper a novel approach inspired by human vision was proposed for biometric images identification. This method consists of a combination of a new image coding based on frequency signals and a highly brain microcircuits inspired neural network architecture, namely liquid state machine. LSM was successfully used in spatio-temporal pattern recognition, which motives the use of a different coding of images being introduced to the reservoir. Three different databases was used to evaluate the efficiency of the proposed system: the ORL face database, the PolyU palmprint database and finally the GPDS off-line 
signatures database. Many experiments were conducted on the three galleries using high frequencies images and low frequencies images in LSM simulations. Then a successful decision level fusion algorithm approach was applied resulting with significant recognition rates that show the reliability of the proposed biometric system.

\section{REFERENCES}

[1] W. S. McCulloc and W. Pitts, "A logical calculus of the ideas immanent in neurons activity," Bulletin of Mathematical Biophysics, vol. 52, pp. 115-133, 1943.

[2] R. J. Schalkoff, "Neural Pattern Recognition," in Pattern Recognition: Statistical, Structural and Neural Approaches, New York, USA: John Wiley \& Sons, 1991.

[3] H. Jaeger, "The "echo state" approach to analyzing and training recurrent neural networks," Bonn, Germany: German National Research Center for Information Technology GMD Technical Report, vol. 148, no. 34, 2001.

[4] W. Maass, T. Natschläger, and H. Markram "Real-time computing without stable states: a new framework for neural computation based on perturbations," Neural Computation, vol. 14, no. 11, pp. 2531-2560, 2002, doi: 10.1162/089976602760407955.

[5] J. Kaiser, R. Stal, A. Subramoney, A. Roennau, and R. Dillmann, "Scaling up liquid state machines to predict over address events from dynamic vision sensors," Bioinspiration \& Biomimetic, vol. 12, no. 5, 2017, doi: 10.1088/1748-3190/aa7663.

[6] H. Burgsteiner, M. Kröll, A. Leopold, and G. Steinbauer, "Movement prediction from real-world images using a liquid state machine," Applied Intelligence, vol. 26, no. 2, pp. 99-109, 2007, doi: 10.1007/s10489-006-0007-1.

[7] Y. Zhang, P. Li, Y. Jin, and Y. Choe, “A Digital Liquid State Machine With Biologically Inspired Learning and Its Application to Speech Recognition," in IEEE Transactions on Neural Networks and Learning Systems, vol. 26, no. 11, pp. 2635-2649, Nov. 2015, doi: 10.1109/TNNLS.2015.2388544.

[8] Y. Jin and P. Li, "Performance and robustness of bio-inspired digital liquid state machines," Neurocomputing, vol. 226, pp. 145-160, 2017, doi: 10.1016/j.neucom.2016.11.045.

[9] W. Zhang and P. Li, "Information-theoretic intrinsic plasticity for online unsupervised learning in spiking neural networks," Frontiers in Neuroscience, vol. 13, 2019, doi: 10.3389/fnins.2019.00031.

[10] N. Soures and D. Kudithipudi, "Deep Liquid State Machines With Neural Plasticity for Video Activity Recognition," Frontiers in Neuroscience, vol. 13, 2019, doi: 10.3389/fnins.2019.00686.

[11] B. J. Grzyb, E. Chinellato, G. M. Wojcik, and W. A. Kaminski, "Facial expression recognition based on Liquid State Machines built of alternative neuron models," 2009 International Joint Conference on Neural Networks, 2009, pp. 1011-1017, doi: 10.1109/IJCNN.2009.5179025.

[12] Q. Wang and P. Li, "D-LSM: Deep Liquid State Machine with unsupervised recurrent reservoir tuning," 201623 rd International Conference on Pattern Recognition (ICPR), 2016, pp. 2652-2657, doi: 10.1109/ICPR.2016.7900035.

[13] F. Rheaume, D. Grenier, and É. Bossé, "Multistate combination approach for liquid state machines in supervised spatio-temporal pattern classification," Neurocomputing, vol. 74, pp. 2842-2851, 2011, doi: 10.1016/j.neucom.2011.03.033.

[14] S. Schliebs, E. Capecci, and N. Kasabov, "Spiking neural network for on-line cognitive activity classification based on EEG data," International Conference on Neural Information Processing, pp. 55-62, 2013, doi: 10.1007/978-3-642-42051-1_8.

[15] A. Polepalli, N. Soures, and D. Kudithipudi, "Reconfigurable digital design of a liquid state machine for spatio-temporal data," Proceedings of the 3rd ACM International Conference on Nanoscale Computing and Communication: NaNoCom'16, no. 15, 2016, pp. 1-6, doi: 10.1145/2967446.2967449.

[16] A. Das et al., "Unsupervised heart-rate estimation wearable with liquid states and a probalistic readout," Neural Networks, vol. 99, pp. 134-147, 2018, doi: 10.1016/j.neunet.2017.12.015.

[17] H. Hazan and L. M. Manevitz, "Topological constraints and robustness in liquid state machines," Expert Systems with Applications, vol. 39, no. 2, pp. 1597-1606, 2012, doi: 10.1016/j.eswa.2011.06.052.

[18] S. Luo, H. Guan, X. Li, F. Xue, and H. Zhou, "Improving liquid state machine in temporal pattern classification," 2018 15th International Conference on Control, Automation, Robotics and Vision (ICARCV), 2018, pp. 88-91, doi: 10.1109/ICARCV.2018.8581122.

[19] Y. Zhou, Y. Jin, and J. Ding, "Surrogate-assisted evolutionary search of spiting neural architectures in liquid state machines," Neurocomputing, vol. 406, pp. 12-23, 2020, doi: 10.1016/j.neucom.2020.04.079.

[20] E. Hourdakis and P. Trahanias, "Use of the separation property to derive liquid state machines with enhanced classification performance," Neurocomputing, vol. 107, pp. 40-48, 2013, doi: 10.1016/j.neucom.2012.07.032.

[21] B. Petro, N. Kasabov, and R. M. Kiss, "Selection and Optimization of Temporal Spike Encoding Methods for Spiking Neural Networks," in IEEE Transactions on Neural Networks and Learning Systems, vol. 31, no. 2, pp. 358-370, Feb. 2020, doi: 10.1109/TNNLS.2019.2906158.

[22] Y. Xu, H. Tang, J. Xing, and H. Li, "Spike trains encoding and threshold rescaling method for deep spiking neural networks," 2017 IEEE Symposium Series on Computational Intelligence (SSCI), 2017, pp. 1-6, doi: 10.1109/SSCI.2017.8285427.

[23] S. Nazari and K. Faez, "Spiking pattern recognition using informative signal of image and unsupervised biologically plausible learning," Neurocomputing, vol. 330, pp. 196-211, 2019, doi: 10.1016/j.neucom.2018.10.066.

[24] M. A. Aoun and M. Boukadoum, "Chaotic liquid state machine," International Journal of Cognitive Informatics and Natural Intelligence, vol. 9, no. 4, pp. 1-20, 2015, doi: 10.4018/IJCINI.2015100101.

[25] T. Reed and H. Wechsler, "Spatial/spatial-frequency representations for image segmentation and grouping," Image and Vision Computing, vol. 9, no. 3, pp. 175-193, 1991, doi: 10.1016/0262-8856(91)90012-E.

[26] R. J. Schalkoff, Digital image processing and computer vision, New York, USA: John Wiley \& Sons, 1989.

[27] F. Hourali and S. Gharravi, "An ear recognition method based rotation invariant transformed DCT," International Journal of Electrical and Computer Engineering, vol. 7, no. 5, pp. 2895-2901, 2017, doi: 10.11591/ijece.v7i5.pp2895-2901.

[28] P. Sinha, B. Balas, Y. Ostrovsky, and R. Russell, "Face Recognition by Humans: Nineteen Results All Computer Vision Researchers Should Know About," in Proceedings of the IEEE, vol. 94, no. 11, pp. 1948-1962, Nov. 2006, doi: 10.1109/JPROC.2006.884093.

[29] W. Maass, "Liquid state machine: motivation, theory and applications," Computability in context: computation and logic in the real world, pp. 275-296, 2010, doi: 10.1142/9781848162778_0008.

[30] E. Goodman and D. Ventura, "Spatiotemporal Pattern Recognition via Liquid State Machines," The 2006 IEEE International Joint Conference on Neural Network Proceedings, 2006, pp. 3848-3853, doi: 10.1109/IJCNN.2006.246880.

[31] L. Pape, J. De Gruijl, and M. Weiring, "Democratic liquid state machines for music recognition," Speech, Audio, Image and Biomedical Signal Processing using Neural Networks, pp. 191-215, 2008, doi: 10.1007/978-3-540-75398-8_9. 
[32] D. Zhang, Wai-Kin Kong, J. You, and M. Wong, "Online palmprint identification," in IEEE Transactions on Pattern Analysis and Machine Intelligence, vol. 25, no. 9, pp. 1041-1050, Sept. 2003, doi: 10.1109/TPAMI.2003.1227981.

[33] A. Eleyan and H. Demirel, "PCA and LDA Based Neural Networks for Human Face Recognition," I-Tech Education and Publishing, pp. 93-106, 2007.

[34] J. K. Sing, S. Thakur, D. K. Basu, M. Nasipuri, and M. Kundu, "High-speed face recognition using self-adaptive radial basis function neural networks," Neural Computing and Applications, vol. 18, no. 8, pp. 979-990, 2009, doi: 10.1007/s00521-009-0242-6.

[35] S. Xu, M. Li, and Y. Cui, "A mixed two-dimensional linear discriminate method," TELKOMNIKA Indonesian Journal of Electrical Engineering and Computer Science, vol. 11, no. 6, pp. 3012-3019, 2013, doi: 10.11591/telkomnika.v11i6.2608.

\section{BIOGRAPHIES OF AUTHORS}

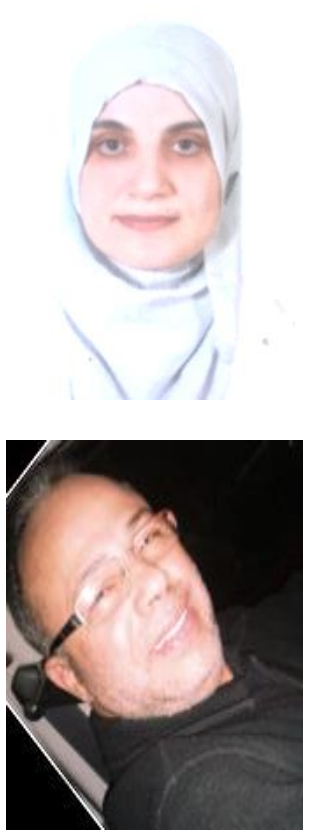

Wassila Boukhari (D) $8 \mathrm{SC}$ S is a Phd student and Associate Professor at University of Sciences and Technology of Oran Mohamed Boudiaf USTO-MB, Algeria. She Holds a Master degree in Computer Science with specialization in pattern recognition and artificial intelligence. Her research areas are image/signal processing, biometrics, pattern recognition, artificial neural networks. She can be contacted at email: wassila.boukhari@univ-usto.dz and boukhari.wassila@gmail.com.

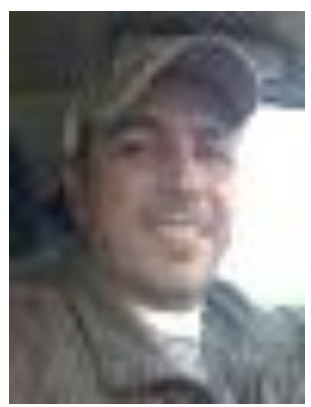

Abderrahim Belmadani (iD) $\mathrm{SC}$ P is a Professor at University of Sciences and Technology of Oran Mohamed Boudiaf USTO-MB, Algeria. His research area are data processing and computer science, modeling and simulation, optimimization and data analysis. He can be contacted at email: abderrahim.belmadani@gmail.com. 